PROCEEDINGS OF THE

AMERICAN MATHEMATICAL SOCIETY

Volume 131, Number 2, Pages 501-509

S 0002-9939(02)06681-9

Article electronically published on June 12, 2002

\title{
APPLICATIONS OF PHASE PLANE ANALYSIS OF A LIÉNARD SYSTEM TO POSITIVE SOLUTIONS OF SCHRÖDINGER EQUATIONS
}

\author{
JITSURO SUGIE AND NAOTO YAMAOKA \\ (Communicated by Carmen C. Chicone)
}

\begin{abstract}
This paper deals with semilinear elliptic equations in an exterior domain of $\mathbb{R}^{N}$ with $N \geq 3$. Sufficient conditions are obtained for the equation to have a positive solution which decays at infinity. The main result is proved by means of a supersolution-subsolution method presented by Noussair and Swanson. By using phase plane analysis of a system of Liénard type, a suitable positive supersolution is found out. Asymptotic decay estimation on a solution of the Liénard system gains a positive subsolution. Examples are given to illustrate the main result.
\end{abstract}

\section{INTRODUCTION}

We consider the semilinear elliptic equation

$$
\Delta u+f(x, u)=0, \quad x \in \Omega,
$$

where $\Omega$ is an exterior domain of $\mathbb{R}^{N}$ with $N \geq 3$, that is, $G_{a}=\left\{x \in \mathbb{R}^{N}:|x|>a\right\} \subset$ $\Omega$ for some $a>0$. Throughout this paper, we assume that $f(x, u)$ is nonnegative and locally Hölder continuous with exponent $\alpha \in(0,1)$ in $\bar{M} \times \bar{J}$ for every bounded domain $M \subset \Omega$ and for every bounded interval $J \subset \mathbb{R}$.

It is well known that de Broglie's wave function

$$
\psi(x, t)=\exp \left(-\frac{i E t}{\hbar}\right) v(x)
$$

is a solution of the Schrödinger equation for a free particle of mass $m$, momentum $p$ and kinetic energy $E$ :

$$
i \hbar \frac{\partial}{\partial t} \psi=-\frac{\hbar^{2}}{2 m} \Delta \psi
$$

where $\hbar=h / 2 \pi$ ( $h$ is Planck's constant) and

$$
v(x)=A \exp \left(\frac{i(p \cdot x)}{\hbar}\right) \text {. }
$$

Received by the editors September 19, 2001.

2000 Mathematics Subject Classification. Primary 35B05, 35J60; Secondary 34C10, 70K05.

Key words and phrases. Positive solution, Schrödinger equation, exterior domain, Liénard system.

The first author was supported in part by Grant-in-Aid for Scientific Research 11304008. 
This equation is generalized into the Schrödinger equation with the potential $V$ and the nonlinearity

$$
i \hbar \frac{\partial}{\partial t} \psi=-\frac{\hbar^{2}}{2 m} \Delta \psi+V(x) \psi-g(x,|\psi|) \psi .
$$

If it has standing waves solutions of the form

$$
\psi(x, t)=\exp \left(-\frac{i E t}{\hbar}\right) u(x)
$$

then the function $u(x)$ must satisfy the elliptic equation

$$
\Delta u+\frac{2 m}{\hbar^{2}}(E-V(x)) u+g(x,|u|) u=0,
$$

which is of the form (1). In quantum mechanics, such are called stationary Schrödinger equations.

The aim of this paper is to give sufficient conditions under which equation (1) has a positive solution in an exterior domain of $\mathbb{R}^{N}$.

For a bounded domain $M \subset \Omega$, let $C^{2+\alpha}(\bar{M})$ denote the usual Hölder space. For simplicity, $C_{\text {loc }}^{2+\alpha}(\Omega)$ is defined as the set of all functions $u: \Omega \rightarrow \mathbb{R}$ such that $u \in C_{\text {loc }}^{2+\alpha}(\bar{M})$ for every bounded domain $M \subset \Omega$. A function $u \in C_{\text {loc }}^{2+\alpha}(\Omega)$ is called a solution of (1) in $\Omega$ if it satisfies equation (1) at every point $x \in \Omega$. Similarly, a function $u \in C_{\text {loc }}^{2+\alpha}(\Omega)$ is called a supersolution (resp., subsolution) of (1) in $\Omega$ if it satisfies the inequality $\Delta u+f(x, u) \leq 0$ (resp., $\geq 0$ ) at every point $x \in \Omega$.

A typical example of (1) is the Emden-Fowler equation

$$
\Delta u+p(x) u^{\gamma}=0, \quad x \in \Omega,
$$

where $p(x)$ is nonnegative and locally Hölder continuous in $\Omega$ and $\gamma$ is a positive number. From this fact, equation (1) is often discussed under a sublinear or a superlinear hypothesis. For instance, equation (1) is said to be sublinear (resp., superlinear) if there exists a $\gamma$ with $0<\gamma<1$ (resp., $\gamma>1$ ) such that $f(x, u) / u^{\gamma}$ is nonincreasing (resp., nondecreasing) in $u$ for each fixed $r=|x|>0$.

Many studies have been made on the existence of a positive solution of (1) in the linear case, the sublinear case and the superlinear case (see [2, 4, 5, 6, 7]). In this paper, we intend to examine another case in addition to these cases. For example, consider the case that

$$
f(x, u)=p(x)\left(u+\frac{u}{4(\log u)^{2}}\right)
$$

for all sufficiently small $u$. Then equation (1) is neither sublinear nor superlinear (of course, equation (1) is not linear). In fact, differentiating $f(x, u) / u^{\gamma}$, we have

$$
\frac{d}{d u}\left(\frac{f(x, u)}{u^{\gamma}}\right)=\frac{p(x)}{u^{\gamma}}\left\{(1-\beta)+\frac{1-\beta-2 / \log u}{4(\log u)^{2}}\right\} \text {. }
$$

Hence, if $0<\gamma<1$ (resp., $\gamma>1$ ), then $f(x, u) / u^{\gamma}$ is increasing (resp., decreasing) for $u>0$ sufficiently small. In the case (2), for any $k>1$, there exists a positive interval $I$ such that

$$
p(x) u<f(x, u)<k p(x) u
$$

for all $x \in \Omega$ and $u \in I$. Hence, from this point of view, we may say that equation (1) is almost linear in such cases as (2). 
For sublinear Schrödinger equations, Swanson [7, Theorem 2.4] gave the following sufficient condition for the existence of a positive solution under the assumption that

$$
0 \leq f(x, u) \leq u \varphi(|x|, u)
$$

for all $x \in \Omega$ and $u>0$, where $\varphi(r, u)$ is locally Hölder continuous with exponent $\alpha \in(0,1)$ and nonincreasing in $u$ for each fixed $r>0$.

Theorem A. Under the assumption (3), equation (1) has a positive solution in an exterior domain if

$$
\int^{\infty} r \varphi(r, c) d r<\infty
$$

for some $c>0$.

Consider the case that $f(x, u)=u / 4|x|^{\beta}$ with $\beta \geq 2$. Then assumption (3) is satisfied with $\varphi(r, u)=1 / 4 r^{\beta}$. Since

$$
\int^{\infty} r \varphi(r, c) d r=\int^{\infty} \frac{1}{4 r^{\beta-1}} d r
$$

for any $c>0$, condition (4) is satisfied if $\beta>2$, but it does not hold if $\beta=2$. Hence, Theorem A is inapplicable to the case $\beta=2$. However, the equation

$$
\Delta u+\frac{u}{4|x|^{2}}=0
$$

has a positive solution, because its radial solutions are represented as the form of

$$
u(x)= \begin{cases}\left(K_{1}+K_{2} \log |x|\right)|x|^{-1 / 2} & \text { if } N=3, \\ K_{3}|x|^{z}+K_{4}|x|^{2-N-z} & \text { if } N \geq 4,\end{cases}
$$

where $K_{i}(i=1,2,3,4)$ are arbitrary constants and $z$ is the root of $z^{2}+(N-2) z+$ $1 / 4=0$.

Assumption (3) is not compatible with the superlinear case and the almost linear case. Hence, instead of (3), we assume that

$$
0 \leq f(x, u) \leq \frac{h(u)}{|x|^{2}}
$$

for all $x \in \Omega$ and $u \geq 0$, where $h(u)$ is locally Lipschitz continuous and positive for $u>0$, and $h(0)=0$. We also prepare the following notation to present a theorem which can be applied to these cases. Write

$$
L_{1}(u)=1, \quad L_{n+1}(u)=L_{n}(u) l_{n}(u), \quad n=1,2, \cdots,
$$

where

$$
l_{1}(u)=2|\log u|, \quad l_{n+1}(u)=\log \left\{l_{n}(u)\right\},
$$

and set

$$
S_{n}(u)=\sum_{k=1}^{n} \frac{1}{\left\{L_{k}(u)\right\}^{2}} .
$$

Define $e_{0}=1$ and $e_{n}=\exp \left(e_{n-1}\right)$. Then we have

$$
l_{n+1}(u)=\log \left\{l_{n}(u)\right\}>0 \quad \text { for } 0<u<1 / \sqrt{e_{n}},
$$


and therefore, the function sequences $\left\{L_{n}(u)\right\},\left\{l_{n}(u)\right\}$ and $\left\{S_{n}(u)\right\}$ are well-defined for $u>0$ sufficiently small. To take some concrete forms of $S_{n}(u)$, for $u>0$ sufficiently small,

$$
\begin{aligned}
& S_{1}(u)=1 \\
& S_{2}(u)=1+\frac{1}{4(\log u)^{2}} \\
& S_{3}(u)=1+\frac{1}{4(\log u)^{2}}+\frac{1}{4(\log u)^{2}(\log (2|\log u|))^{2}}
\end{aligned}
$$

and so on.

Our main result is stated in the following:

Theorem 1. Assume (5) and suppose that there exists a positive integer $n$ such that

$$
\frac{h(u)}{u} \leq \frac{(N-2)^{2}}{4} S_{n}(u)
$$

for all $u>0$ sufficiently small. Then equation (1) has a positive solution $u(x)$ in an exterior domain with $\lim _{|x| \rightarrow \infty} u(x)=0$.

\section{A supersolution AND A SUBSOlution}

We will prove the main result by use of the so-called "supersolution-subsolution" method. The lemma below yields from a result of Noussair and Swanson [5, Theorem 3.3].

Lemma 2. If there exists a positive supersolution $\bar{u}$ of (1) and a positive subsolution $\underline{u}$ of $(1)$ in $G_{b}$ such that $\underline{u}(x) \leq \bar{u}(x)$ for all $x \in G_{b} \cup C_{b}$, where $b \geq a$ and $C_{b}=\left\{x \in \mathbb{R}^{N}:|x|=b\right\}$, then equation (1) has at least one solution $u$ satisfying $u(x)=\bar{u}(x)$ on $C_{b}$ and $\underline{u}(x) \leq u(x) \leq \bar{u}(x)$ through $G_{b}$.

To apply Lemma 2, we have to find a suitable positive supersolution of (1) and a positive subsolution of (1) which is not greater than the supersolution. For this purpose, we consider the nonlinear differential equation

$$
\frac{d^{2}}{d r^{2}} w+\frac{N-1}{r} \frac{d}{d r} w+\frac{1}{r^{2}} g(w)=0, \quad r>a
$$

where $g(w)$ satisfies a suitable smoothness condition for the uniqueness of solutions of the initial value problem and the signum condition

$$
w g(w)>0 \text { if } w \neq 0 .
$$

Then we have the following nonoscillation theorem for equation (7).

Lemma 3. Assume (8). If there exists a positive integer $n$ such that

$$
\frac{g(w)}{w} \leq \frac{(N-2)^{2}}{4} S_{n}(|w|)
$$

for $w>0$ or $w<0,|w|$ sufficiently small, then all nontrivial solutions of (7) are nonoscillatory.

Proof. Using phase plane analysis of a Liénard system, Sugie et al. [10, Lemma 3.2] have proved that under the assumption (8), all nontrivial solutions of the equation

$$
\frac{d^{2}}{d r^{2}} w+\frac{2}{r} \frac{d}{d r} w+\frac{1}{r^{2}} g(w)=0
$$


are nonoscillatory if

$$
\frac{g(w)}{w} \leq \frac{1}{4} S_{n}(|w|)
$$

for $w>0$ or $w<0,|w|$ sufficiently small. Hence, the lemma is true for $N=3$.

Suppose that $N \geq 4$. Let

$$
\tau=(N-2) r^{N-2} \quad \text { and } \quad v(\tau)=w(r) .
$$

Then equation (7) becomes

$$
\frac{d^{2}}{d \tau^{2}} v+\frac{2}{\tau} \frac{d}{d \tau} v+\frac{1}{\tau^{2}} g^{*}(v)=0
$$

where $g^{*}(v)=g(v) /(N-2)^{2}$. This equation has the form of (10). It follows from (9) that

$$
\frac{g^{*}(w)}{w}=\frac{g(w)}{(N-2)^{2} w} \leq \frac{1}{4} S_{n}(|w|)
$$

for $w>0$ or $w<0,|w|$ sufficiently small, that is, (11) is satisfied with $g(w)=g^{*}(w)$. Hence, by Lemma 3.2 in [10] again, we see that all nontrivial solutions of (7) are nonoscillatory in the case $N \geq 4$.

By virtue of Lemma 3, we can choose a solution of (7) which is eventually positive. In the next section, we will show that the positive solution is a supersolution of (1). To get a positive subsolution of (1), we need to estimate the asymptotic behavior of positive solutions of (7) as follows.

Lemma 4. Assume (8) and (9). Then there exist a positive number $b \geq a$ and $a$ positive solution $w(r)$ of $(7)$ such that $\lim _{r \rightarrow \infty} w(r)=0$

$$
b^{N-2} w(b) \leq r^{N-2} w(r) \quad \text { for } r \geq b .
$$

Proof. From Lemma 3 we see that equation (7) has a positive solution. Let $w(r)$ be the positive solution. Then there exists a $b \geq a$ such that

$$
w(r)>0 \text { for } r \geq b .
$$

The change of variables $r=e^{s}$ and $w(r)=\xi(s)$ transforms equation (7) into the Liénard system

$$
\begin{aligned}
& \frac{d}{d s} \xi=\eta-(N-2) \xi, \\
& \frac{d}{d s} \eta=-g(\xi) .
\end{aligned}
$$

Let $(\xi(s), \eta(s))$ be the solution of (12) corresponding to $w(r)$. Then we have

$$
\xi(s)>0 \quad \text { for } s \geq \log b .
$$

By (8) we obtain

$$
\frac{d}{d s} \eta(s)<0 \text { for } s \geq \log b .
$$

It is well known that the zero solution of (12) is globally asymptotically stable (for example, see [1, [3, [8]). Hence, we conclude that the solution $(\xi(s), \eta(s))$ tends to the origin as $s \rightarrow \infty$. This means that $w(r)$ approaches zero as $r \rightarrow \infty$. 
We will show that $\eta(s) \geq 0$ for $s \geq \log b$. Suppose that $\eta\left(s_{0}\right)<0$ for some $s_{0} \geq \log b$. Then, by (12)-(14) we have

$$
\frac{d}{d s} \xi(s)<\frac{d}{d s} \xi(s)+(N-2) \xi(s)=\eta(s) \leq \eta\left(s_{0}\right)
$$

for $s \geq s_{0}$. Integrate this inequality from $s_{0}$ to $s$ to obtain

$$
\xi(s)<\xi\left(s_{0}\right)+\eta\left(s_{0}\right)\left(s-s_{0}\right) \rightarrow-\infty \quad \text { as } s \rightarrow \infty .
$$

This is a contradiction to (13).

Since $\eta(s) \geq 0$ for $s \geq \log b$, we see that

$$
\frac{d}{d s} \xi(s) \geq-(N-2) \xi(s) \quad \text { for } s \geq \log b .
$$

Hence, integrating the both sides, we have

$$
b^{N-2} \xi(\log b) \leq e^{(N-2) s} \xi(s) \text { for } s \geq \log b,
$$

namely, $b^{N-2} w(b) \leq r^{N-2} w(r)$ for $r \geq b$. Thus, the lemma is proved.

We are now ready to prove the main theorem.

\section{Proof of the main theorem}

Consider the nonlinear differential equation

$$
\frac{d^{2}}{d r^{2}} w+\frac{N-1}{r} \frac{d}{d r} w+\frac{1}{r^{2}} h^{*}(w)=0, \quad r \geq a,
$$

where $a$ is the number given in (1) and

$$
h^{*}(w)=\left\{\begin{array}{cc}
h(w) & \text { for } w \geq 0, \\
-h(-w) & \text { for } w<0 .
\end{array}\right.
$$

Then, from assumption (5) we see that $h^{*}(w)$ satisfies the signum condition (8), and therefore, equation (15) is in the type of (7). Also, by condition (6) we have

$$
\frac{h^{*}(w)}{w} \leq \frac{1}{4} S_{n}(|w|)
$$

for $w>0$ and $w<0,|w|$ sufficiently small. Hence, from Lemma 3 we conclude that all nontrivial solutions of (15) are nonoscillatory. For this reason, we can choose a solution $w(r)$ which is positive for all $r \geq b$ with some $b \geq a$ (we may regard $b$ as the positive number in Lemma 4). As in the proof of Lemma 4, we can show that $w(r)$ approaches zero as $r$ tends to $\infty$. Note that $w(r)$ is also a positive solution of the equation

$$
\frac{d^{2}}{d r^{2}} w+\frac{N-1}{r} \frac{d}{d r} w+\frac{1}{r^{2}} h(w)=0 .
$$

Let $\bar{u}$ be the function defined in $G_{b}$ by $\bar{u}(x)=w(r), r=|x| \geq b$. Then, by assumption (5) we obtain

$$
\begin{aligned}
\Delta \bar{u}(x)+f(x, \bar{u}(x)) & =\frac{d^{2}}{d r^{2}} w(r)+\frac{N-1}{r} \frac{d}{d r} w(r)+f(x, w(r)) \\
& \leq \frac{d^{2}}{d r^{2}} w(r)+\frac{N-1}{r} \frac{d}{d r} w(r)+\frac{1}{|x|^{2}} h(w(r)) \\
& =\frac{d^{2}}{d r^{2}} w(r)+\frac{N-1}{r} \frac{d}{d r} w(r)+\frac{1}{r^{2}} h(w(r))=0 .
\end{aligned}
$$


Hence, $\bar{u}$ is a supersolution of (1) in $G_{b}$. We next denote $\underline{u}(x)=b^{N-2} w(b) /|x|^{N-2}$ for $|x| \geq b$. Then, since $f(x, u)$ is nonnegative, we get

$$
\begin{aligned}
\Delta \underline{u}(x)+f(x, \underline{u}(x)) & \geq \frac{d^{2}}{d r^{2}}\left(\frac{b^{N-2} w(b)}{r^{N-2}}\right)+\frac{N-1}{r} \frac{d}{d r}\left(\frac{b^{N-2} w(b)}{r^{N-2}}\right) \\
& =\frac{(N-2)(N-1) b^{N-2} w(b)}{r^{N}}-\frac{N-1}{r} \frac{(N-2) b^{N-2} w(b)}{r^{N-1}}=0 .
\end{aligned}
$$

This means that $\underline{u}(x)$ is a subsolution of $(1)$ in $G_{b}$.

From Lemma 4 we see that

$$
\underline{u}(x)=\frac{b^{N-2} w(b)}{|x|^{N-2}}=\frac{b^{N-2} w(b)}{r^{N-2}} \leq w(r)=\bar{u}(x)
$$

for $|x| \geq b$. Hence, by means of Lemma 2, we conclude that there exists a positive solution $u(x)$ of (1) satisfying $\underline{u}(x)=u(x)=\bar{u}(x)$ on $C_{b}$ and $\underline{u}(x) \leq u(x) \leq \bar{u}(x)$ through $G_{b}$. Since $w(r)$ tends to zero as $r \rightarrow \infty$, the positive solution $u(x)$ also tends to zero as $|x| \rightarrow \infty$. This completes the proof.

\section{Discussion}

To illustrate the main theorem, we will give some examples which are the almost linear case. One cannot apply previous results on the existence of a positive solution to those examples. For brevity, we define the function $\phi(u ; \lambda)$ by $\phi(0 ; \lambda)=0$ for any $\lambda \geq 0$ and

$$
\phi(u ; \lambda)= \begin{cases}u+\frac{\lambda u}{(\log |u|)^{2}} & \text { for } 0<u \leq \frac{1}{e}, \\ (3 \lambda+1) u-\frac{2 \lambda}{e} & \text { for } u>\frac{1}{e} .\end{cases}
$$

Then it is easy to check that $\phi(u ; \lambda)$ is continuous for $u \geq 0$ and is continuously differentiable for $u>0$.

We first consider the elliptic equation

$$
\Delta u+p(x) \phi(u ; 1 / 4)=0
$$

in an exterior domain $\Omega$ of $\mathbb{R}^{N}$ with $N \geq 3$. Let

$$
f(x, u)=p(x) \phi(u ; 1 / 4)
$$

and

$$
h(u)=\frac{(N-2)^{2}}{4} \phi(u ; 1 / 4) .
$$

Then condition (5) holds and condition (6) is satisfied with $n=2$. Hence, as a direct consequence of Theorem 1, we have the following result.

Example 5. If

$$
0 \leq p(x) \leq \frac{(N-2)^{2}}{4|x|^{2}}
$$

for $x \in \Omega$, then equation (16) has a decaying positive solution.

Let us take another example to show how sharp Theorem 1 is. For this purpose, we restrict $p(x) /|x|^{2}$ to any constant. 
Example 6. Consider the equation with two parameters

$$
\Delta u+\frac{\mu}{|x|^{2}} \phi(u ; \lambda)=0
$$

instead of (16). Then, from Theorem 1 we have the following conclusions:

(i) if $0 \leq \mu<(N-2)^{2} / 4$, then equation (17) has a decaying positive solution for all $\lambda \geq 0$

(ii) if $\mu=(N-2)^{2} / 4$, then equation (17) has a decaying positive solution for $0 \leq \lambda \leq 1 / 4$.

Proof. Let $f(x, u)=\mu \phi(u ; \lambda) /|x|^{2}$ and $h(u)=\mu \phi(u ; \lambda)$. Since $\lambda$ and $\mu$ are nonnegative, condition (5) is satisfied. Hence, it is enough to check that condition (6) holds for $u>0$ sufficiently small. If $\lambda=0$, then $h(u) / u=\mu \leq(N-2)^{2} / 4$ for all $u>0$, that is, condition (6) is satisfied with $n=1$. We assume that $\lambda$ is positive.

(i) We can choose an $\varepsilon_{0}>0$ so small that $\mu\left(1+\varepsilon_{0}\right)<(N-2)^{2} / 4$. For any $\lambda>0$, we see that

$$
\frac{h(u)}{u}=\mu\left(1+\frac{\lambda}{(\log u)^{2}}\right)<\mu\left(1+\varepsilon_{0}\right)<\frac{(N-2)^{2}}{4}
$$

for $0<u<\exp \left(-\sqrt{\lambda / \varepsilon_{0}}\right)$. Hence, condition (6) is satisfied with $n=1$.

(ii) In this case, we have

$$
\frac{h(u)}{u}=\mu\left(1+\frac{\lambda}{(\log u)^{2}}\right) \leq \frac{(N-2)^{2}}{4}\left(1+\frac{1}{4(\log u)^{2}}\right)
$$

for $u$ sufficiently small, namely, condition (6) with $n=2$.

Recently, by use of phase plane analysis of a Liénard system, Sugie et al. 9. Lemma 4.4] have given an oscillation theorem for equation (10) under the assumption (8) as follows.

Theorem B. Assume (8) and suppose that there exists a $\lambda$ with $\lambda>1 / 4$ satisfying

$$
\frac{g(w)}{w} \geq \frac{1}{4}+\frac{\lambda}{(2 \log |w|)^{2}}
$$

for $|w|$ sufficiently small. Then all nontrivial solutions of (10) are oscillatory.

To compare with conclusion (ii) of Example 6, we consider the equation

$$
\Delta u+\frac{(N-2)^{2}}{4|x|^{2}} \phi^{*}(u ; \lambda)=0
$$

where

$$
\phi^{*}(u ; \lambda)=\left\{\begin{array}{cc}
\phi(u ; \lambda) & \text { for } u \geq 0, \\
-\phi(-u ; \lambda) & \text { for } u<0 .
\end{array}\right.
$$

It is clear that $\phi^{*}(u ; \lambda)$ is odd, and therefore, it satisfies the signum condition (8). As shown in Sections 2 and 3, the change of variables

$$
v(\tau)=w(r)=u(x), \quad r=|x| \quad \text { and } \quad \tau=(N-2) r^{N-2}
$$

reduces equation (19) to

$$
\frac{d^{2}}{d \tau^{2}} v+\frac{2}{\tau} \frac{d}{d \tau} v+\frac{1}{4 \tau^{2}} \phi^{*}(v ; \lambda)=0
$$


This is of the form (10). Since

$$
\frac{\phi^{*}(v ; \lambda)}{4 v}=\frac{1}{4}+\frac{\lambda}{(2 \log |v|)^{2}}
$$

for $|v|$ sufficiently small, from Theorem B it turns out that if $\lambda>1 / 4$, then equation (19) fails to have positive radial solutions. Hence, together with the second conclusion in Example 6, we see that equation (19) has a positive radial solution if and only if $\lambda \leq 1 / 4$.

\section{REFERENCES}

[1] T. A. Burton, On the equation $x^{\prime \prime}+f(x) h\left(x^{\prime}\right) x^{\prime}+g(x)=e(t)$, Ann. Mat. Pura Appl., 85 (1970), 277-285. MR 41:7201

[2] A. Constantin, Positive solutions of Schrödinger equations in two-dimensional exterior domains, Monatsh. Math., 123 (1997), 121-126. MR 97i:35026

[3] J. R. Graef, On the generalized Liénard equation with negative damping, J. Differential Equations, 12 (1972), 34-62. MR 48:6542

[4] E. S. Noussair and C. A. Swanson, Positive solutions of semilinear Schrödinge equations in exterior domains, Indiana Univ. Math. J., 28 (1979), 993-1003. MR 81b:35031

[5] E. S. Noussair and C. A. Swanson, Positive solutions of quasilinear elliptic equations in exterior domains, J. Math. Anal. Appl., 75 (1980), 121-133. MR 81j:35007

[6] C. A. Swanson, Bounded positive solutions of semilinear Schrödinger equations, SIAM J. Math. Anal., 13 (1982), 40-47. MR 83c:35032

[7] C. A. Swanson, Criteria for oscillatory sublinear Schrödinger equations, Pacific J. Math., 104 (1983), 483-493. MR 84c:35008

[8] J. Sugie, D.-L. Chen and H. Matsunaga, On global asymptotic stability of systems of Liénard type, J. Math. Anal. Appl., 219 (1998), 140-164. MR 99c:34111.

[9] J. Sugie, K. Kita and N. Yamaoka, Oscillation constant of second order nonlinear self-adjoint differential equations, to appear in Ann. Mat. Pura Appl. (4).

[10] J. Sugie, N. Yamaoka and Y. Obata, Nonoscillation theorems for a nonlinear self-adjoint differential equation, Nonlinear Anal., 47 (2001), 4433-4444.

Department of Mathematics and Computer Science, Shimane University, Matsue 6908504, JAPAN

E-mail address: jsugie@math.shimane-u.ac.jp

Department of Mathematics and Computer Science, Shimane University, Matsue 6908504, JAPAN

E-mail address: yamaoka@math.shimane-u.ac.jp 